Journal of Computer Science 6 (12): 1524-1530, 2010

ISSN 1549-3636

(C) 2010 Science Publications

\title{
Three-Dimension Coronary Artery Tree Curvature Confirmation
}

\author{
${ }^{1}$ Hassan H. Khalil, ${ }^{1}$ Rahmita O.K. Rahmat, ${ }^{2}$ D.M. Zamrin, \\ ${ }^{1}$ Ramlan Mahmod and ${ }^{1}$ Norwati Mustapha \\ ${ }^{1}$ Department of Computer Science and Information Technology, \\ University Putra Malaysia, 43400, Serdang, Malaysia \\ ${ }^{2}$ Department of Surgery, Heart and Lung Centre, National University of Malaysia Medical Centre, \\ HUKM, 56000 Cheras, Kuala Lumpur, Malaysia
}

\begin{abstract}
Problem statement: Three-Dimension (3D) reconstruction is one of the vital and robust tools that provide aid in many fields, especially medicine. This article is about 3D shape similarity and it presents a comparison approach between principal curvature methods of 3D output. Our approach follows the concept of using the gray scale value as the $\mathrm{z}$ dimension and the other approach is a standard one. A comparison of the curvature of the 3D outputs will be made between the standard approach and our proposed one to prove its correctness. We propose to use the standard deviation technique to compare the output features of the 3D coronary artery trees. We applied a standard approach of 3D shape similarity and compared the features with ours. The standard approach was published in 1998 as a study comparing certain 3D curvature measurement algorithms. Approach: Our approach consists of three major steps: (1) Apply the paraboloid fitting technique from the standard approach; (2) Apply the 3D reconstruction algorithm proposed in this research on the same data in step (1) and (3) Apply the Standard Deviation technique on both outputs from (1) and (2) and compare the outputs. Results: Experimental evaluation has been done on clinical raw data sets where the experimental results revealed that both outputs are totally matched. Conclusion: The match in the output refers to the correctness of the proposed 3D output and subsequently its coronary artery tree curvature as well.
\end{abstract}

Key words: Angiograms, coronary artery, 3D reconstruction, 3D curvature, 3D model of coronary artery

\section{INTRODUCTION}

Recent advances in the area of computer graphics have enabled a synthesis of photo-realistic images from virtual scene descriptions that comprise many graphical systems. The synthesized images are often used for replacing real photographs, or for the representation of imaginary scenes that do not actually exist in the real world. There are many cases where the reality of the images being synthesized is highly dependent on the quality of models being used. This motivates the development of high quality models that are able to describe even fine details of objects. Although there are various types of scanning devices available including 3D laser scanners, the development of high quality models is still considered a difficult and time consuming task. Therefore, most designers are willing to explore and reuse the existing models that have been developed by others instead of creating their own models from scratch. Fortunately, there are several archives and collections existing on the Internet that provide 3D models in particular. However, these archives are rarely accompanied by suitable search functions in order to compare and match shapes of stored models. The system designers are usually forced to set aside considerable time for investigation of the models one by one (Krsek et al., 1998).

The fact that shape features are invariant under three dimensional rigid motions and the scaling strategy are two important issues for many 3D processing operations on object shapes, including segmentation and shape recognition and matching (Gotoda, 2003). There have been recent advances in 3D technology for shape acquisition that require the development of reliable methods for the processing of range data (Gotoda, 2003). Surface creases that are defined through principal curvature extrema are usually independent in terms of scale and view and, therefore,

Corresponding Author: Hassan H. Khalil, Department of Computer Science and Information Technology, University Putra Malaysia, 43400, Serdang, Malaysia 
there are numerous applications of them in geometric modeling and image processing as well as in other fields (Gotoda, 2003). Differential parameters including normal, principal curvature, principal direction and others can be utilized in 3D computer vision to solve a variety of basic tasks including the following.

Segmentation: The initial set point is divided into a number of subsets that have the same geometric (or other) features (Douros and Buxton, 2002).

Surface classification: The segmented points could be categorized as belonging to a given surface type, for example, concave or convex, cylindrical or planar or spherical and others, (Douros and Buxton, 2002).

Surface reconstruction: There are differential parameters that can help in finding the parameters representing the best-fit surface, in a more accurate way than the direct way of using the measured points (Douros and Buxton, 2002).

Registration: Differential parameters show invariant properties under Euclidean transformations, making them useful in algorithms for surface registration (Douros and Buxton, 2002).

Therefore, the algorithms that can compute differential parameters are very important. It must be noted that the idealized separation of the above tasks might be an over simplification. For example, there are differential parameters that can be estimated better from a fitted surface, however, surface fitting requires segmentation to be performed first and is usually driven by estimates of differential parameters. Here, an important issue is about the stability of the applied estimation methods as well as their dependency on noise from the input data (Douros and Buxton, 2002). The computing of 3D surface curvature from its discrete point form has become an essential task in many applications including feature recognition, segmentation and rendering. Extruding the 3rd Cartesian dimension (z) from the gray scale value was proposed by us to render a coronary artery tree cloud of points. The 3D reconstruction algorithm is summarized in this study. It was a vital task to prove that the proposed use of the gray scale value as the z-dimension is true and that it gives the correct 3D surface curvature.

The detecting of salient extrema of the principal curvatures, the ridges and ravines, in range data was proposed by (Belyaev and Anoshkina, 2005) by combining methods of classical differential geometry with modern image processing techniques and developing a coordinate-independent computational procedure.

Furthermore, (Douros and Buxton, 2002) indicated that the high-order surface based method estimates the curvature by locally fitting a surface.

A novel approach to 3D shape comparison was presented by (Gotoda, 2003). The approach is imagebased on the similarity of images representing the appearance of shapes in various perspectives. The method was proposed to compare 3D shapes but it could not be applied to the comparison of very complex shapes.

Our method and the method in (Krsek et al., 1998) are similar in that both use an implicit z-dimension function to compute the 3D surface curvature. In this study, we employ an implicit z-dimension function defined by (Krsek et al., 1998) and we compare it with our 3D shape rendering, which will be presented later in the study. The paraboloid fitting is the kind of shape close to the heart according to the physicians and its cross-sections comes close to similarity.

Other methods and approaches were proposed by (Yoshimi and Tomita, 1994; Shum et al., 1996; Zhang and Fiume, 2002) and focused on the 3D shape similarity in different ways.

Yoshimi and Tomita (1994), they proposed a new robust method for calculating curvature vectors from the data range. Meanwhile (Shum et al., 1996) studied the 3D shape similarity between closed surfaces. They presented a curved or polyhedral 3D object of genus zero using a mesh representation that has nearly uniform distribution with known connectivity among mesh nodes.

Finally, (Zhang and Fiume, 2002) proposed a novel method to normalize the Fourier Descriptor (FD) of a 3D contour with respect to two of its FD coefficients that correspond to the lowest non-zero frequencies.

None of the above algorithms and approaches or others that we studied presented 3D shape similarity of medical images. Our study depends on the medical images called angiograms. This kind of image indicates the heart's vessels in gray scale. The closest technique to ours was the one presented in (Douros and Buxton, 2002) as mentioned above.

The proposed approach here is for $3 \mathrm{D}$ shape comparison. The approach compares geometric features using the Standard Deviation (STD) technique. The STD will represent the geometric features of the 3D shape. Since the method is STD-based, it can handle almost any kind of shape that is renderable. Furthermore, it enables us to find a similarity between a pair of shapes that can be mapped in 3D. The main aim 
of this approach is the proof and confirmation of the 3D output's curvature which an algorithm has already done. In that algorithm we proposed a technique for 3D reconstruction of the coronary artery tree called Fast 3D (F3D) and used the gray scale value as the z-dimension. This is helpful, especially when dealing with morphologically similar shapes, such as coronary artery trees of the same species. The approach can cover a wider class of similarity than other methods, plus it is fast.

\section{MATERIALS AND METHODS}

Here, we will describe in detail our approach to compare 3D shape features and prove that our F3D has provided the correct 3D shape. We will explain our F3D algorithm in detail and then explain the approach of Paraboloid Fitting as in (Douros and Buxton, 2002).

F3D reconstruction: This algorithm, which is about fast 3D coronary artery reconstruction has already been developed by us. The steps of this algorithm are: (1) Vessel extraction; (2) Data files and (3) 3D reconstruction.

Vessel extraction: The dynamic movement of coronary artery tree vessels have been very difficult to study through the use of normal angiograms. The clinical evidence shows that vessel extraction is a very useful process for surgical and clinical study. From Hoover et al. (2000), we improved the algorithm to use its vessel extraction but with angiogram input instead of retina images. The improvement was to set the value of $\sigma$ in the Gaussian function in the range $1 \leq \sigma \leq 2$. This proposed improvement over Hoover's provided good results for coronary artery tree vessel extraction. We are going to use the output from this step and input it into our $3 \mathrm{D}$ reconstruction algorithm. The output from this step will be the extracted vessels in $2 \mathrm{D}$. The vessels will be in gray scale (1-256) and the background is black. Figure 1 shows an example of the extracted vessels angiograms. Figure 2 is the flowchart for the 3D reconstruction algorithm.

Data files: The extracted images of the vessels in the last step are in 2D and with gray scale vessels and black background. In this phase we will try to save the data of those images, i.e., save the $\mathrm{x}, \mathrm{y}$ and $\mathrm{z}$ coordinates. The $\mathrm{x}$ and $y$ are the rows and columns of the images, but what about the $\mathrm{z}$ value? In this study we suggest a move that is not new but has not been used with angiograms or $3 \mathrm{D}$ reconstruction. We use the gray scale value of the vessel as the $\mathrm{z}$ coordinate of our $3 \mathrm{D}$ system, as in Eq. 1 and Fig. 3. The data (x, y, z) is saved in data files, i.e., a data file for each angiogram of the extracted vessels. The purpose of this step is that we can use any one of the data files to construct an executional file that can be used to reconstruct the 3D shape of any of the extracted vessels angiograms afterwards. Randomly we choose any of the data files and we project it to a $\mathrm{VC}++$ and OPENGL library. The output of this library will be an executional file that we save for the later stages.

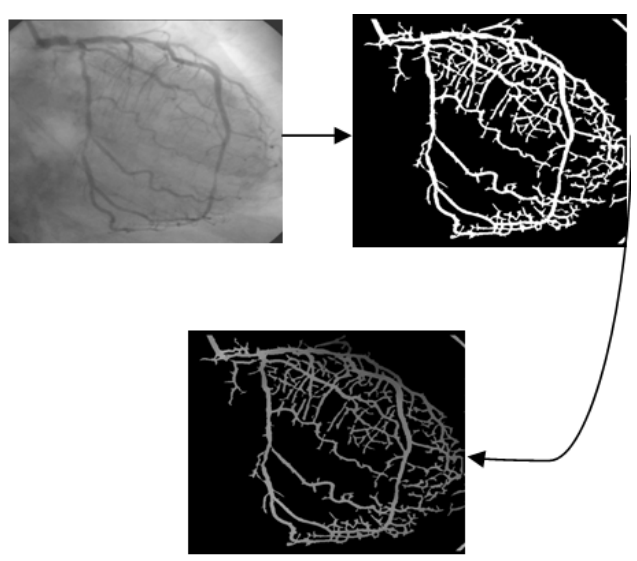

Fig. 1: Extracted vessels in gray scale values

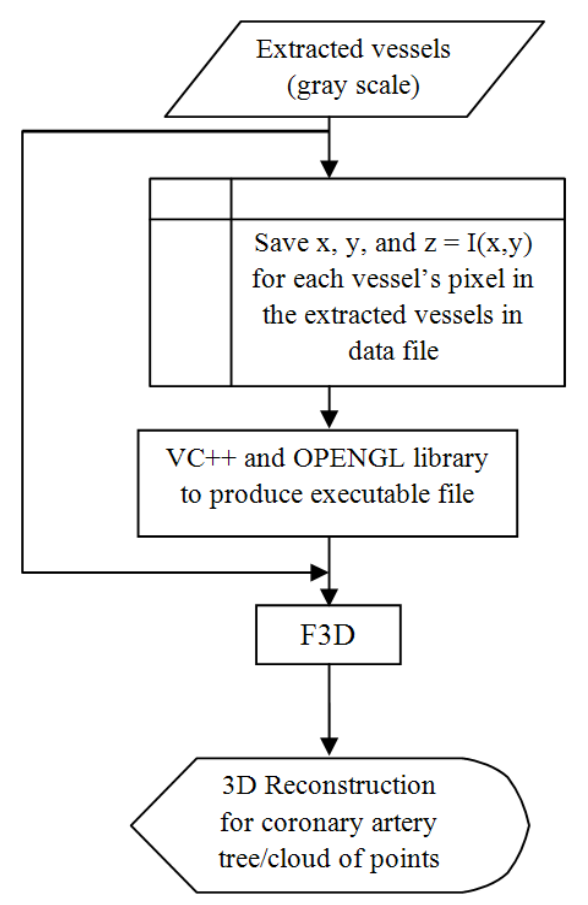

Fig. 2: Flowchart of 3D reconstruction algorithm 

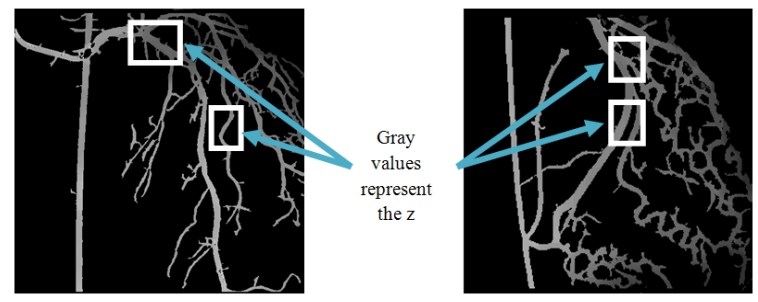

Fig. 3: $\mathrm{Z}$ values of the extracted vessels

3D reconstruction: After completing the extraction and data file steps we are able to complete the process by reconstructing our 3D shape. Having already saved the angiograms of the extracted vessels and the executional file from the last step we are able to build exceptional software called Fast 3 Dimension (F3D). To reconstruct the 3D shape from any extracted vessels angiogram, we project the angiogram plus the executional file that was built from the last step to the F3D software. This can reconstruct the 3D shape of that coronary artery tree in the specific angiogram in a few seconds. The 3D shape will be clear enough to see any side of it and it can be rotated through any angle we request.

Using F3D, we can control the speed of rotation, zoom in and out and change the color of the shape to fit with the user's eyes. F3D will generate the shape as points projected in the 3D space such that each point represents a pixel in the extracted vessels of the coronary artery with its $\mathrm{x}, \mathrm{y}$ and $\mathrm{z}$ dimensions. The software was provided with a paint service that allows the user to show part of the coronary artery tree or omit the tiny vessels from the 3D shape. The paint button can be pressed and the angiogram can be edited accordingly.

By the end of the fourth step of the algorithm, the 3D shape of any coronary artery tree can be obtained and we have the ability to enhance and develop the tree according to the needs of the study.

Paraboloid fitting: this method is on the basis of surface approximation by a second order analytic surface. A standard approach comprises several steps (Douros and Buxton, 2002).

Estimation of the normal: Is by local approximation of the data by a plane.

Transformation of the neighborhood points: Into a coordinate system that has its origin at a given point and also $+\mathrm{z}$ along the normal.
Fitting a second order surface: In the above coordinate system of $\mathrm{z}=\mathrm{f}(\mathrm{x}, \mathrm{y})$.

Computation of curvature: From the parameters belonging to the analytic surface.

To do this, the data should form a single valued function within a small neighborhood of each point. The algorithm's implementation can eliminate the first two steps. The data will be approximated directly through a paraboloid with its axis parallel to the $\mathrm{z}$ axis of the measuring machine. Therefore, it can be guaranteed that the input data is in the form of a function $\mathrm{z}=\mathrm{f}(\mathrm{x}, \mathrm{y})$, as long as it is obtained via a physical range finder that is from a single view. The computation results are obtained faster and are comparable with fitting in local coordinates. The second order surface that is fitted can be given by (Douros and Buxton, 2002):

$z=a x^{2}+b x y+c y^{2}+d x+e y+f$

Where:

A $\quad=[\mathrm{a}, \mathrm{b}, \mathrm{c}, \mathrm{d}, \mathrm{e}, \mathrm{f}]$ include the paraboloid parameters

$[\mathrm{x}, \mathrm{y}, \mathrm{z}]=$ The surface point coordinates

Now we consider the approach that we created to compare the 3D shape features of the Paraboloid Fitting (PF) and the F3D reconstruction. The comparison will be made by using standard deviation, which can provide feature similarity or the difference between two 3D shapes of the same object (coronary artery tree in the case). The algorithm has the following steps:

1. Calculate and save data files (x,y,z) of F3D's output

2. Calculate and save data files $(x, y, z)$ of PF's output

3. Calculate the Standard Deviation (STD) of data files from (1) and (2) above

4. From (3), we compare the (STD) of both (1) and (2)

5. If the STD of both outputs are similar then it can be said that the F3D output is correct and both 3D shapes have the same features, otherwise they do not

Figure 4 shows the flowchart of the 3D shape similarity. The approach was tested on a database of 100 angiogram pictures and a sample of the results will be shown in the experimental results. After the vessel extraction step, we save the vessels' extracted images to use them for both F3D and PF algorithms later. 
Applying F3D and PF will produce a 3D output of the coronary artery trees, i.e., $\mathrm{x}, \mathrm{y}$ and $\mathrm{z}$ dimensions that can be saved and used in the STD calculation. The last step of the method concerns the calculation of the STD from the data files that have already been saved.

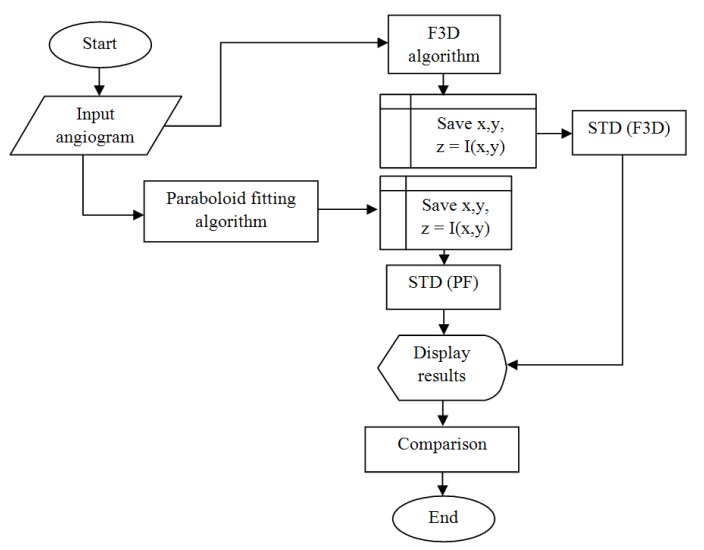

Fig. 4: 3D shape similarity flowchart

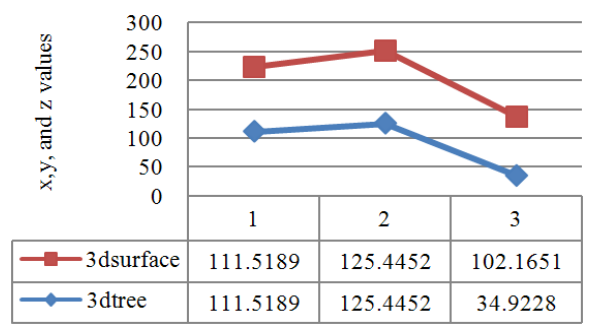

(a)

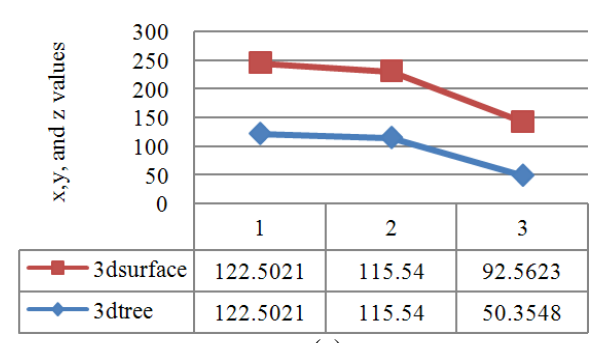

(c)

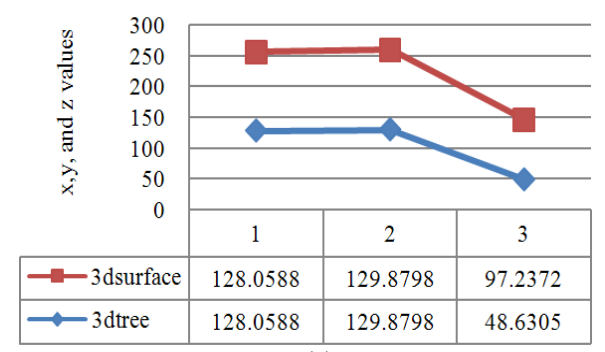

(e)
We used MATLAB to calculate the STD. For each 3D output we will obtain a STD with three values for each dimension. Subsequently, we will draw those values and compare the figure of STD of both 3D outputs. PF is a standard algorithm that was applied and published in (Douros and Buxton, 2002) therefore, the comparison will be used to prove the correction of F3D.

\section{RESULTS}

We tested the approach on clinical real angiograms for different patients. The range of image sizes was $500 \times 500$ pixels. We applied F3D first and then PF and we calculated STD for both of them. Table 1 below shows the STD results for both F3D and PF for 10 angiograms from the data set and Fig. 5 shows the diagrams of those results. From the results given in Table 1 and Fig. 5, we can see that the STD, which represents the geometric features of the 3D shape, has the same diagram. This means that the 3D shapes of both the F3D and PF have the same geometric features.

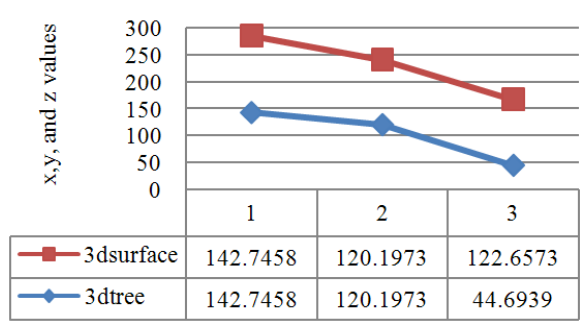

(b)

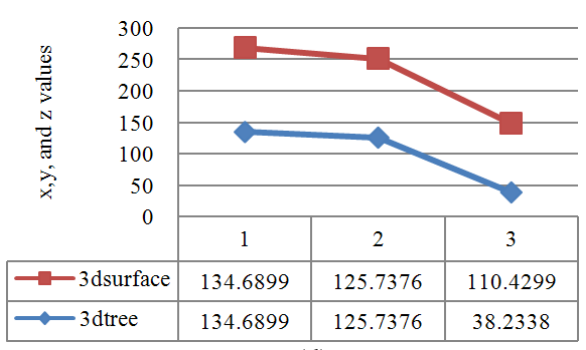

(d)

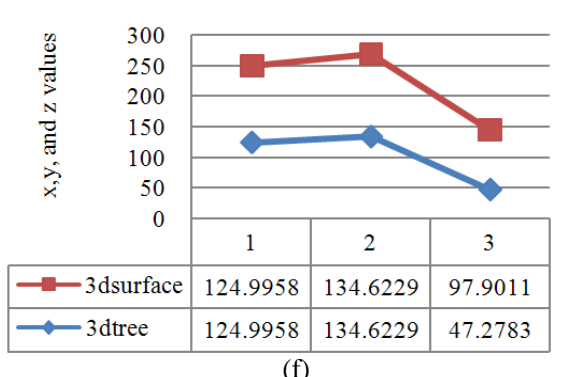



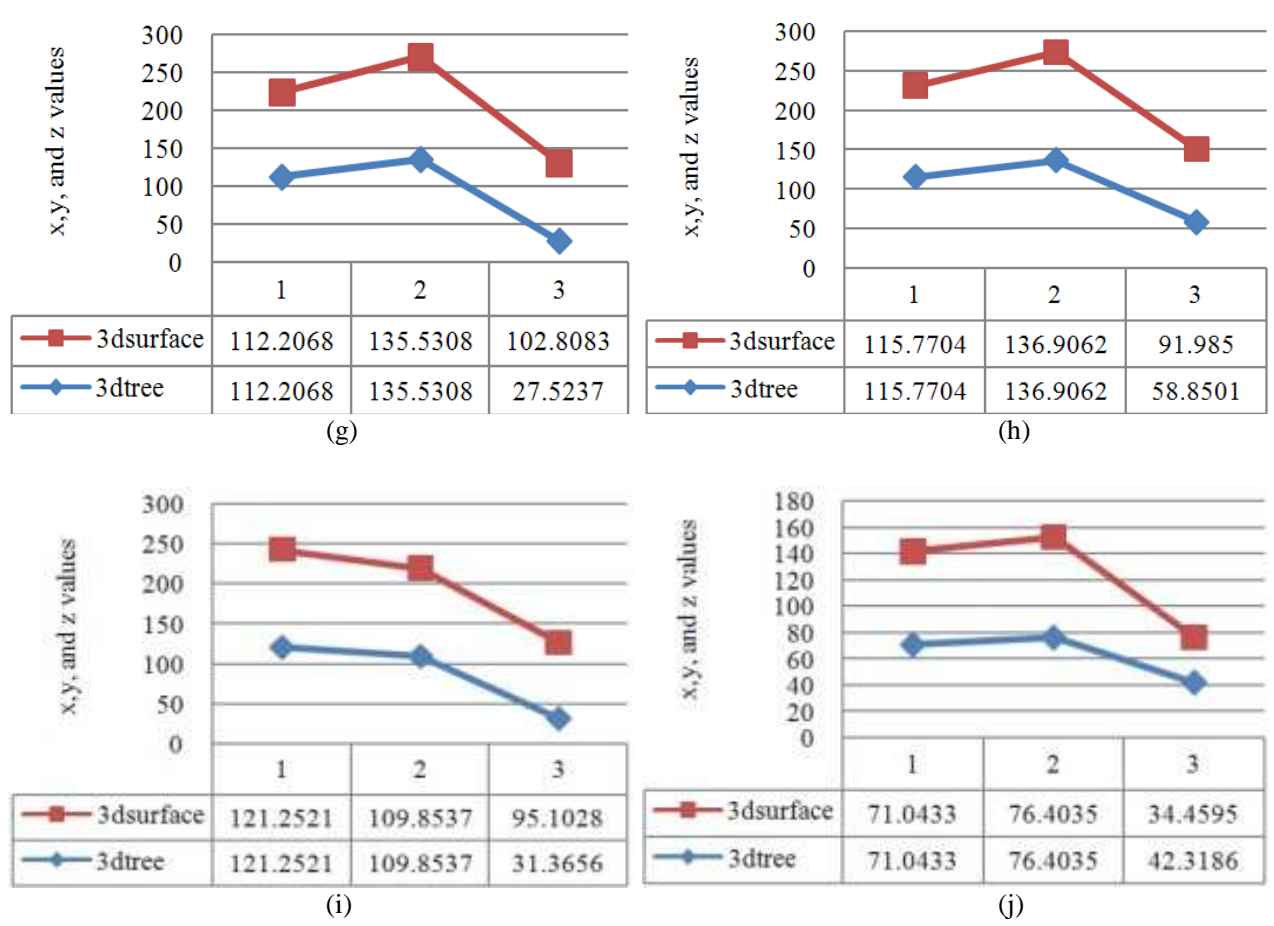

Fig. 5: STD diagrams of the ten angiogram images arranged according to Table 1

\begin{tabular}{llrr}
\multicolumn{4}{l}{ Table 1: STD results of both F3D and PF for 10 images } \\
\hline Images & Dimension & STD/F3D & STD/PF \\
\hline Image 1 & $\mathrm{X}$ & 111.5189 & 111.5189 \\
& $\mathrm{Y}$ & 125.4452 & 125.4452 \\
Image 2 & $\mathrm{Z}$ & 34.9228 & 102.1651 \\
& $\mathrm{X}$ & 142.7458 & 142.7458 \\
& $\mathrm{Y}$ & 120.1973 & 120.1973 \\
Image 3 & $\mathrm{Z}$ & 44.6939 & 122.6573 \\
& $\mathrm{X}$ & 122.5021 & 122.5021 \\
& $\mathrm{Y}$ & 115.5400 & 115.5400 \\
Image 4 & $\mathrm{Z}$ & 50.3548 & 92.5623 \\
& $\mathrm{X}$ & 134.6899 & 134.6899 \\
& $\mathrm{Y}$ & 125.7376 & 125.7376 \\
Image 5 & $\mathrm{Z}$ & 38.2338 & 110.4299 \\
& $\mathrm{X}$ & 128.0588 & 128.0588 \\
& $\mathrm{Y}$ & 129.8798 & 129.8798 \\
Image 6 & $\mathrm{Z}$ & 48.6305 & 97.2372 \\
& $\mathrm{X}$ & 124.9958 & 124.9958 \\
& $\mathrm{Y}$ & 134.6229 & 134.6229 \\
Image 7 & $\mathrm{Z}$ & 47.2783 & 97.9011 \\
& $\mathrm{X}$ & 112.2068 & 112.2068 \\
Image 8 & $\mathrm{Y}$ & 135.5308 & 135.5308 \\
& $\mathrm{Z}$ & 27.5237 & 102.8083 \\
Image 9 & $\mathrm{X}$ & 115.7704 & 115.7704 \\
& $\mathrm{Y}$ & 136.9062 & 136.9062 \\
& $\mathrm{Z}$ & 58.8501 & 91.9850 \\
Image 10 & $\mathrm{X}$ & 121.2521 & 121.2521 \\
& $\mathrm{Y}$ & 109.8537 & 109.8537 \\
& $\mathrm{Z}$ & 31.3656 & 95.1028 \\
& $\mathrm{Y}$ & 71.0433 & 71.0433 \\
& $\mathrm{Z}$ & 76.4035 & 76.4035 \\
& & 42.3186 & 34.4595 \\
\hline
\end{tabular}

\section{DISCUSSION}

The proposed approach here is to compare 3D shapes and show the similarity between them. The approach used the STD technique to represent the geometric features. This approach is used to prove the correctness of the F3D algorithm, as discussed above. F3D for 3D coronary tree reconstruction uses the gray scale value as its z-dimension and an approach had to be created to prove that F3D provides the correct curvature of the $3 \mathrm{D}$ coronary artery tree. The idea to come up with this approach was inspired by (Krsek et al., 1998). We applied one of the algorithms (PF) from that article which was considered similar to F3D. They used a different $\mathrm{z}$ dimension formula for paraboloid fitting. We applied their algorithm and F3D and calculated the geometric features for each one. Subsequently, we put those features in diagrams and compared them to achieve the goal from this approach. The results indicate that $\mathrm{F} 3 \mathrm{D}$ provides the correct output and subsequently the true coronary artery tree curvature. We used MATLAB to calculate the STD. The diagrams were drawn in Excel application. The approach is vital for determining 3D shape similarity and could be applied to different 3D shapes and other image types. 


\section{CONCLUSION}

The 3D similarity has been a serious issue in the research field, however, although are many approaches in this field, to date, there is no comprehensive approach we could depend on to solve our problem in this study. Our study involves objects that have been extracted from medical images and to prove the correctness of the 3D shape curvature we need to prove the correctness of its $3 \mathrm{D}$ reconstruction. In this study a new approach has been proposed for comparing 3D coronary artery tree features with a standard technique that has already been approved and published. The basic idea of our approach is to use the STD technique to compare the 3D features of our 3D output with the standard 3D Paraboloid shapes. The results have shown that around $95 \%$ of our 3D shapes matched the features of the standard Paraboloid approach. The approach was robust to prove the correctness of 3D shape similarity and as a result the correctness of our 3D shapes.

\section{REFERENCES}

Belyaev, A. and E. Anoshkina, 2005. Detection of surface creases in range data. Lecture Notes Comput. Sci., 3604: 50-61. DOI: 10.1007/11537908_4

Douros, I. and B.F. Buxtonm, 2002. Three-dimensional surface curvature estimation using quadric surface patches. University College London. http://eprints.ucl.ac.uk/156231/
Gotoda, H., 2003. 3D shape comparison using multiview images. NII J., 7: 19-25. http://www.nii.ac.jp/journal/pdf/07/07-03.pdf

Hoover, A.D., V. Kouznetsova and M. Goldbaum, 2000. Locating blood vessels in retinal images by piecewise thresholdprobing of a matched filter response. IEEE Trans. Med. Imag., 19: 203-210. DOI: $10.1109 / 42.845178$

Krsek, P., G. Lukacs and R.R. Martin, 1998. Algorithms for computing curvatures from range data. Inform. Geomet., 3: 1-16.

Shum, H.Y., M. Hebert and K. Ikeuchi, 1996. On 3D shape similarity. Proceedings of the IEEE Computer Society Conference on Computer Vision and Pattern Recognition, June 18-20, IEEE Xplore Press, San Francisco, CA., USA., pp: 526-531. DOI: $10.1109 /$ cvpr.1996.517122

Yoshimi, T. and F. Tomita, 1994. Robust curvature vectors calculation from range data using ISL method. MVA, 94: 506-509.

Zhang, H. and E. Fiume, 2002. Shape matching of 3D contours using normalized Fourier descriptors. Proceedings of the Shape Modeling International Meeting, May 17-22, IEEE Xplore Press, Banff, Alta., Canada, pp: 261-268. DOI: 10.1109/SMI.2002.1003554 